\title{
Las aportaciones de la prensa de proximidad a la formación de comunidades locales. Veintidós años de Levante de Castelló
}

\author{
Ramón S. PARdo BALdeóN \\ Universitat Jaume I de Castelló \\ pardor@uji.es
}

\begin{abstract}
Resumen
La prensa local desempeña un papel destacado como vertebrador de las comunidades locales en las que se inserta y a las que dota de un relato propio. El suministro de información de servicio y la organización y participación en actividades socioculturales ayuda a reforzar la identidad de los lectores alrededor de un espacio social. Levante de Castelló contribuyó a formar y cohesionar una comunidad provincial en torno a identidades propias en las que lo cercano, lo identificable con su cultura, es prioritario. Sus informaciones constituyeron el soporte cultural de esas comunidades.
\end{abstract}

Palabras clave: Periodismo local, comunidades locales, información de servicio, vertebración, Levante de Castelló

The proximity press imputs to the local communities trainning. Twenty two years of journalism in Levante de Castelló

\begin{abstract}
The local press plays an important role as the backbone of the local communities in which it take part and in which it gives a unique tale. The supply of service information, plus the organization and participation in the socio-cultural activities helps to reinforce the reader's identity surrounding social space. Levante de Castelló contributed to train and draw together a provincial community around self-identities where the closeness, the undetectable of their culture, was the priority. Their news established the cultural support of those communities.
\end{abstract}

Key Words: Local press, local communities, service information, backbone, Levante de Castelló.

\section{Referencia normalizada:}

Pardo Baldeón, R. S. (2013) Las aportaciones de la prensa de proximidad a la formación de comunidades locales. Veintidós años de Levante de Castelló. Historia y Comunicación Social. Vol. 18. № Especial Diciembre. Págs. 105-114.

Sumario: 1. Introducción. 1.1. Metodología. 2. La prensa cercana estimula la identidad local. 3. El papel de Levante de Castelló en la creación de una comunidad local (1991-2012). 4. Conclusiones. 5. Bibliografía. 5.1. Recursos web 


\section{Introducción}

La prensa de proximidad ha cumplido y cumple una función tan importante en el sistema democrático como es la vertebración de la sociedad en la que se inserta a partir de los mensajes que elabora. En plena sociedad de la información, con unos ciudadanos sometidos al constante bombardeo de informaciones alejadas geográficamente de su entorno, la prensa local permite a los ciudadanos seguir el pulso de lo que acontece en su entorno más próximo y tener una idea clara de la agenda de la actualidad local. De ahí que el suministro de esas informaciones cercanas cobra un papel vital a la hora de conformar la opinión pública a partir de una realidad social que los mismos medios se encargan de recrear. Con ello están contribuyendo a dinamizar las sociedades y generar sinergias identitarias al ejercer como foro de encuentro y puesta en común de valores que afectan a un entorno geográfico y social determinado. Esta función unificadora de los medios de comunicación se dio durante el período de Transición democrática española y en el posterior proceso de constitución y asentamiento de las comunidades autónomas.

Fruto del papel asumido por los medios de comunicación es la eclosión vivida por la prensa local durante las dos últimas décadas del pasado siglo. En ese contexto histórico, y amparados en su función de servicio público a los ciudadanos/lectores, los diarios locales contribuyeron a la formación y consolidación de comunidades locales vertebradas a partir de un relato propio elaborado por los medios de comunicación. Esto fue posible a partir de la política desarrollada por los medios y tendente a crear cauces de participación de los usuarios que requerían su implicación, según López (2005: 2).

Dos características sobresalen en el periodismo local, vinculadas ambas con su servicio público, de un lado la capacidad de facilitar información a los ciudadanos, a partir de las informaciones publicadas, y de otro la de creación de comunidades, faceta ésta alrededor de la cual gira este trabajo. Comunidades que se agrupan en torno a temas o ideas que les unen e identifican. Tarea ésta que ejercen los medios locales y que les permite agrupar a los ciudadanos y hacerles sentirse integrantes de un territorio físico concreto. Un espacio que va desde el que atañe a una ciudad hasta el comarcal o provincial, según los casos. Para cumplir esa misión es preciso que el medio se implique en la actividad de ese colectivo al que sirve. Implicación que puede acometer bien desde el desarrollo de su papel de agente propiciador del debate necesario para formar la opinión pública o desde el de impulsor u organizador de actividades socioculturales o deportivas encaminadas a lograr un maridaje con sus lectores, de los que depende la viabilidad del producto. Es en esta fase de dinamización social cuando el diario deja de ser un medio frío de comunicación con su audiencia para meterse en el interior de sus lectores y buscar aquellos temas que les interesan para satisfacer su demanda. Algo que le lleva, en ocasiones, a organizar eventos que calan en la audiencia.

El papel desarrollado desde 1991 por el diario Levante de Castelló puede servir de referencia a la hora de analizar el éxito de la prensa local como herramienta capaz de 
aglutinar colectivos sociales para construir comunidades, en este caso provinciales, en torno a un espacio no sólo físico/geográfico, sino ideológico, cultural. Un espacio/ territorio al que el periódico aporta un relato propio, relato que convierte en parte de la historia de la provincia, que ha coprotagonizado en estos 22 años, un tiempo durante el que se estaba forjando la identidad cultural y lingüística valenciana desde el poder autonómico radicado en Valencia (Martínez Sanchis, 2007: 43). En estos años, el diario ha sido fiel a los principios de sus editores de difundir y apostar por el papel que desempeñan administraciones e instituciones locales como vertebradoras del territorio, lo que permitió aglutinar a los ciudadanos en torno a unos usos culturales y hacerles partícipes de un territorio común, para reforzar ese sentimiento de comunidad.

\subsection{Metodología}

El objeto del trabajo es analizar como la prensa provincial española ha cumplido en los años posteriores a la Transición democrática su papel de dinamizadora y creadora de comunidades a su alrededor, adoptando el papel de institución referencial para su público, en la misma medida que la prensa estatal contribuyó a cohesionar la esfera pública. En Castellón, los diarios de referencia despertaron a las élites políticas y al pueblo que unieron fuerzas para reivindicar ante Valencia mejoras de infraestructuras de todo tipo. Unas demandas que tuvieron en los diarios de mayor tirada un vehículo de expresión que favoreció la formación de la opinión pública. Levante de Castelló cumplió esa función de servicio público que tienen los medios como difusores y dinamizadores de la cultura, las tradiciones, la lengua y la realidad social e institucional del entorno en el que se mueven, sin olvidarse de promover el pluralismo y la participación de los grupos sociales. En definitiva ser ese espejo de la realidad sociocultural e institucional capaz de "fomentar la cohesión social y la pertenencia territorial" (Calvo, 2013).

Para acometer el trabajo se optó por aplicar el estudio de casos, con especial énfasis en el análisis de contenidos y en las entrevistas en profundidad a dos de los delegados de Levante de Castelló, Vicent Borja, en el inicio de la andadura del medio en marzo de 1991, y el actual, José Beltrán, que ha vivido el proceso de implantación del medio desde su primer número. De forma simultánea se realizó una selección de 160 informaciones sobre temas relevantes que generaron debate ciudadano y que fueron recogidas en el diario entre marzo de 1991 y diciembre de 2012. Textos que relatan una serie cambios políticos (en el consistorio de Castelló y en la diputación provincial) y de infraestructuras (soterramiento de la vía férrea o apertura del campus universitario). Cambios que tuvieron repercusión en la sociedad. 


\section{La prensa cercana estimula la identidad local}

Que la prensa es uno de los pilares que garantizan la democracia, siempre que cumpla su función de control de las acciones de los políticos (Casero-Ripollés, 2009), faceta que complementa con la de agente activo fruto de la mediatización (Casero-Ripollés, 2008: 113) no ofrece dudas, como tampoco las genera el que la prensa local contribuye a crear, estimular y reforzar las señas de identidad del territorio en el que se inserta (López, 2005: 1; De Fontcuberta, 1997: 48). Si bien no es menos cierto que para cumplir ese cometido los medios locales se implican y participan en las actividades de esa comunidad. De Fontcuberta (1997) y Xambó (2001) sostienen que los medios de proximidad aportan a las comunidades que ayudan a consolidar elementos tales como la identidad regional, la vertebración y la potenciación de los rasgos culturales propios. Por ello, estos medios tienen una percepción más real de lo que ocurre en su entorno y de lo que le interesa a sus usuarios, vinculada a su faceta de servicio. Función que han de compaginar con la de satisfacer el ansia de su audiencia por conocer todo lo que pasa al lado de su casa, en su barrio o su trabajo. Razón por la que los diarios locales han orientado sus criterios informativos hacia la oferta de contenidos más prácticos y de utilidad para sus lectores, lo que se denomina prensa de servicios (Izquierdo, 2010; López y Macià, 2007).

Lograr que sus lectores sean activos, no meros espectadores pasivos de lo que ocurre a su alrededor, es la base del éxito de los medios locales y para ello convierten, al periódico en este caso, en un foro de papel en el que se debaten los asuntos que les interesan y afectan. La prensa logra reforzar así su función de espejo de la realidad que refleja una cultura o identidad cercana de la comunidad a la que representa (Esteve y Fernández del Moral, 1995). Una comunidad con la que el periódico interactúa a través de las informaciones que selecciona en un intento de lograr que los ciudadanos/lectores se vean reflejados en sus páginas (López, Galindo y Villar, 1998). En ese contexto, las informaciones de proximidad se constituyen en un referente fundamental para el enriquecimiento de la diversidad cultural y más en unos años marcados por la sociedad de la información y el efecto globalizador de internet (Moragas, 1998). Ese interés por lo próximo que alimenta la prensa local se refleja en un sondeo que corrobora las preferencias informativas de los españoles por lo que ocurre en su entorno o su país más que por las noticias internacionales (Fundación BBVA, 2013: 11). Algo en lo que no se alejan de las respuestas que se dan en el resto de países europeos sondeados por la fundación.

La prensa local se ha convertido en la institución de referencia a la que recurren los ciudadanos a la hora de encontrar su norte. Es esa brújula que les da las coordenadas precisas que les ayudan a tomar posición sobre aquellos asuntos que les afectan. El periódico les da esa identidad que genera siempre una opinión sólida y que evita que existan tantas opiniones divergentes como habitantes formen esa comunidad social. Tan dispares serán como carentes de análisis y elementos argumentativos. Y lo hace desde una activa implicación social que facilita crear esa vinculación identitaria entre 
periódico y público como agentes integrantes de un mismo territorio y una misma comunidad social.

Así, además de su compromiso fundamental con la información y servicio a su público, la prensa local asume el de contribuir a esa vertebración social que precisan las sociedades para tomar conciencia de grupo o comunidad. Una responsabilidad que emana del hecho de que estos medios, con sus informaciones analíticas y de contextualización, interpretan la realidad social, dotándola de sentido (Fernández Beltrán y Vilar, 2002: 238-239). Y es que los diarios locales informan de las actividades y decisiones que adoptan las administraciones para trasladárselas a los ciudadanos, con el fin de legitimarlas, además de para contribuir a formar su opinión (López y Macià, 2007: 33).

Uno de los valores que debe preservar un rotativo local para cumplir su papel de agente vertebrador y dinamizador de la sociedad es el de garantizar la pluralidad informativa y más si quiere servir como herramienta modeladora de la comunidadad, a la que ha de ayudar a preservar la identidad local, a fin de mantener viva la personalidad de la provincia y la ciudad, como recoge Nos Aldás (2001: 480-481).

\section{El papel de Levante de Castelló en la creación de una comunidad local (1991- 2012)}

Los medios de comunicación social castellonenses tienen algunas peculiaridades que los diferencian de otros que también se editan para un ámbito provincial. Y es que Castellón siempre ha mirado de reojo hacia Valencia, epicentro de la política en la comunidad. Un recelo que se puso de manifiesto durante el proceso de construcción autonómica coincidente con la expansión de los periódicos locales. Los castellonenses tenían conciencia de comunidad, pero no tenían los cauces a través de los cuales mostrarse como comunidad de intereses socioculturales sólidos, con sus administraciones dependientes en exceso de las decisiones políticas que se adoptaban en Valencia. Por ello, la llegada de Levante de Castelló en marzo de 1991 con su apuesta por poner en valor las instituciones locales, siguiendo la línea editorial de el grupo Editorial Prensa Ibérica (EPI), de fomentar "la vinculación de los contenidos de sus cabeceras a los intereses generales de los territorios donde se editan" (Laguna, 1992: 203) abrió una ventana a los castellonenses que tuvieron ocasión de exponer sus ideas amparados en el pluralismo que impulsó el diario. Por su perfil más progresista, Levante se granjeó el favor de un sector importante de la sociedad que buscaba una alternativa al oficialismo de Mediterráneo. Sin embargo, pese a la disparidad de sus líneas editoriales, ambos medios lograron que los castellonenses tomasen conciencia como comunidad provincial y se aliasen para defender sus intereses ante las administraciones autonómica y estatal.

Y es que cada sociedad tiene unos mecanismos de respuesta común ante las situaciones que vive y los retos que afronta, por ello sus miembros responden con conduc- 
tas basadas en los referentes culturales que les dotan de identidad. Una identidad que se construye a partir de las informaciones que reciben desde los medios y que conforman la realidad de lo qué ocurre en el entorno. Por ello, la prensa, y en especial la local, tiene la responsabilidad de construir la realidad de la comunidad en la que se inserta, pero esa responsabilidad también conlleva el dotar de sentido a esa realidad.

El diario de Editorial Prensa Ibérica (EPI) fue el primero en poner en valor las instituciones locales castellonenses algunas tan olvidadas como la diputación provincial, al destinar a un redactor a cubrir su día a día, moviendo a sus rivales a hacer lo propio, rompiendo poco a poco el monopolio informativo que tenía el ayuntamiento capitalino. Sin embargo no fue este el único cambio que trajo Levante de Castelló a la ciudad, porque a través de las informaciones aparecidas en sus páginas ayudó a los castellonenses a darse cuenta de la necesidad de dotarse de infraestructuras tanto culturales como de comunicación. En el primer caso apoyó el impulso al campus universitario de la Universitat Jaume I y a ésta como institución social para fomentar el debate y favorecer el relanzamiento de la ciudad, mientras que también hizo ver a los castellonenses que la barrera de la vía férrea que partía en dos a la ciudad debía suprimirse y para ello debía realizarse el soterramiento, cuyo proceso expropiatorio no se vio exento de polémica y opiniones divergentes que tuvieron acogida en el foro de papel que es un diario local. La construcción a partir de los artículos periodísticos de una conciencia cívica en la que primaba el desarrollo urbano y la idea del progreso fue decisiva a la hora de que Castelló ganase una trama urbana con la Universidad como referente.

El diario, en su afán de servir a los lectores, también dio cuenta de la gestación de una infraestructura de comunicaciones de gran envergadura como el aeropuerto de Castellón. Sin oponerse frontalmente, elaboró un corpus de informaciones que buscaban de los lectores la reflexión sobre la necesidad de un aeropuerto y el coste-oportunidad del mismo y para ello dio cabida a las posturas de todos los sectores sociales implicados. Al final, el aeropuerto, una obra polémica, está acabado, pero carece de licencia de apertura dos años después de su inauguración oficial. Sin embargo, la reiterada denuncia desde las páginas del diario de la falta de apoyo gubernativo y la implicación a regañadientes de la Generalitat Valenciana, ha generado un importante gasto para construir una infraestructura cuyo futuro es incierto, como lo es también su viabilidad, la que también cuestionó el periódico y los agentes sociales que usaron su tribuna.

Otra infraestructura sobre cuya necesidad, y, sobre todo, su magnitud hizo reflexionar el diario a sus lectores fue el macro proyecto urbanístico de Mundo ilusión, que planteaba el desarrollo de tres millones de metros cuadrados. Un proyecto muy vinculado al aeropuerto y que, pasado el tiempo, aún no ha visto colocada su primera piedra. En este caso, los vecinos expropiados y los colectivos ecologistas, tuvieron en el diario una ventana abierta para exponer sus posturas.

Desde una apuesta por el medioambiente y los valores patrimoniales, Levante de Castelló, se mostró a favor del desarrollo e implementación de un plan de instala- 
ciones medioambientales para tratar los residuos urbanos generados en la provincia, pero alertando de los inconvenientes que supondría la llegada de instalaciones de tratamiento de residuos tóxicos que alterarían el entorno. Este riesgo, que el diario trasladó a los vecinos, fue decisivo para que la comarca de la Plana Alta se movilizara contra una incineradora que se iba a construir en la zona en 2006. Finalmente, la empresa y la administración autonómica desistieron de la iniciativa y, tras paralizar el proyecto en julio de 2006, lo retiraron en julio de 2008. En los primeros quince de días trascurridos entre la segunda quincena de abril y la primera semana de mayo de 2006, el diario generó hasta 18 informaciones, con Cartas al Director incluidas abordando la situación. Todo ello desde el respeto a la pluralidad informativa y el debate que favorece la toma de conciencia ciudadana, que dio lugar a la aparición y desarrollo de movimientos sociales y plataformas ciudadanas.

Levante de Castelló optó por ser una caja de resonancias de todos los movimientos sociales y por servir como escaparate en el que estos colectivos mostraran sus opiniones, logrando aunar intereses aislados en la defensa del territorio o el medioambiente al ofrecerles un punto de encuentro. El diario se convirtió así en una institución de referencia que les aportaba datos a partir de los cuales fijar sus posiciones. Cumpliendo su función de ofertar informaciones/noticias que favorecieran la formación de opinión del público. Todo ello sin olvidar la vocación comarcal del diario, proclive a crear comunidades comarcales con intereses y referentes culturales comunes. Comunidades éstas que veían como en las páginas del periódico de EPI tenían datos de la actualidad que se gestaba en su territorio.

En una comparativa de los años 1991, cuando salió a la calle el primer número de Levante de Castelló, 2001 y 2008 se comprueba, además del paso hacia las primeras páginas de las secciones de Local y Comarcas, como de las 40 noticias de media del ámbito provincial se pasa a las 62, a pesar del inconveniente que supuso la reducción del número de páginas del diario, que pasó de las 82 de media a las 60 . Se ve claramente el peso que la información cercana tiene en el diario, lo que corrobora su interés por vertebrar la provincia en base a crear conciencia de comunidad. Inclusive el hecho de que las viñetas humorísticas de Xipell dieran protagonismo a los actores políticos provinciales también fue una contribución a la familiarización de los castellonenses con sus gestores políticos y a tomar conciencia de qué papel desempeñaban.

Desde el primer momento el diario buscó formar parte de la vida social de Castellón y su provincia y para ello sus responsables no dudaron en traspasar la frontera física que suponen las páginas del diario y organizaron una serie de actividades, con un trasfondo festivo-cultural. Levante de Castelló montó con motivo de las fiestas fundacionales de la ciudad un punto de encuentro de los ciudadanos: la denominada terraza, que pronto sería imitada por otros periódicos e incluida en el acervo ciudadano como algo propio de la cultura festiva. Suya fue también la apuesta por destacar el contenido cultural de este cita festiva con la elaboración de un cuadernillo dedicado íntegramente a divulgar aspectos de la fiestas y las costumbres rodeándose de un variado elenco de representantes de la cultura provincial, desde escritores a diseñadores, pasando por historiadores y pintores. Precisamente éstos últimos fueron 
los encargados de ilustrar las portadas de estos suplementos que han sido objeto de coleccionista. Con el paso del tiempo, tanto las citas festivas en la terraza como el suplemento festivo-cultural se han integrado como un elemento más de las fiestas, como también lo han sido las tradicionales partidas de "pilota" valenciana. El diario favoreció así, con una implicación que trascendió más allá de la información de servicio, la identificación de medio y lectores, acercándolos más hasta hacerlos sentir participes de un territorio concreto.

El hecho de que el diario encontrase un nicho de mercado en un sector de la sociedad, el más progresista, como corrobora Martínez Sanchis (2007), al que le ofrecía un periodismo profesional condicionó la orientación y contenidos del medio para dar satisfacción a la demanda de sus lectores. Ello también propició que fuerzas políticas con responsabilidad de gobierno considerarán al periódico de EPI un medio, sino hostil poco próximo a sus intereses, algo que también marcó las relaciones entre las dos instituciones y que dejó al diario, en ocasiones, sin conocer detalles de las actuaciones del poder provincial que podían interesar a los lectores.

\section{Conclusiones}

Así como los medios de comunicación social de ámbito estatal ayudan a configurar la opinión pública de sus lectores, los periódicos locales contribuyen a la creación de comunidades locales, que se vertebran alrededor de unos referentes socioculturales propios que les dotan de una identidad y una personalidad que cohesiona a esas comunidades. Una identidad en la que lo próximo, lo cercano, juega un papel prioritario y en cuya formación tienen un destacado papel los medios que con sus informaciones les dotan de un relato propio.

Levante de Castelló contribuyó con sus informaciones, en especial con su apuesta comarcal, a vertebrar la provincia, a la que dotó de un relato y personalidad que identifica a los miembros de su comunidad como grupo más allá de los lindes geográficos-administrativos, dado que el vínculo común de sus referentes culturales les cohesiona. En el caso de Castellón la ligazón es la defensa de intereses comunes como la mejora de las infraestructuras: culturales o de comunicaciones y la unidad de acción ante Valencia, sede del poder administrativo y que tradicionalmente había dejado en un segundo plano a la provincia. El medio se implicó en la transformación de la sociedad a partir de la defensa de algunas de esas demandas a la par que logró canalizar las acciones de los ciudadanos, que se aglutinaron en plataformas cívicas cuyas acciones hicieron despertar a los políticos para hacer valer el peso de Castellón en Valencia.

El diario contribuyó a mantener vivos esos valores culturales de la provincia, aquellos que aglutinan a sus audiencias y cohesionan colectivos. Grupos sociales que contribuyó a dinamizar mediante su implicación en la organización de eventos culturales que le acercaban a ellos. 
El periódico jugó ese papel de foro de papel que tienen los medios de comunicación como difusores y multiplicadores del número de receptores de una idea (Anderson, 2006). Ideas con las que ayudan a los ciudadanos y a sus comunidades a moldear su opinión a partir de la interacción con los demás actores sociales (Borrat, 1989: 9). $\mathrm{Y}$ es que el diario se abrió a los agentes sociales, que encontraron en sus páginas un escaparate de pluralidad informativa en el que exponer sus ideas para que los ciudadanos contasen con elementos suficientes para tener opinión. Junto a los otros dos diarios provinciales ha contribuido en la última década del siglo XX y la primera del nuevo a escribir el relato de una comunidad, la sociedad castellonense, una parte de la cual se ve reflejada en las páginas de la segunda cabecera en cuanto a número de lectores de la provincia.

El hecho de que, como se recoge en el trabajo, el diario fuera elegido como fuente de información prioritaria por el sector más progresista de la sociedad castellonense influyó en que la selección de noticias e informaciones, así como la orientación desde las páginas de opinión haya tendido más a satisfacer la demanda de un sector de esa comunidad lo que ha ido en detrimento del resto y del propio diario. Algo que se refleja en los índices de lectura de la OJD, que en 2012 dieron a Mediterráneo una media de 90.000 lectores por los 25.000 de Levante de Castelló, el segundo diario de la provincia.

\section{Bibliografía}

ANDERSON, Benedict (2006): Comunidades imaginadas. Reflexiones sobre el origen y la difusión del nacionalismo. FCE. México.

BELTRÁN, Adolf (2009): La sociedad valenciana y los medios de comunicación. Zeitschrifft für Katalanistik, 22 Barcelona

BORRAT, Héctor (1989): El periódico, actor político. Gustavo Gilli. Barcelona.

CASERO RIPOLLÉS, Andreu (2008): "Modelos de relación entre periodistas y políticos. La perspectiva de la negociación constante". En: Estudios sobre el Mensaje Periodístico, 14, 111-128.

CASERO RIPOLLÉS, Andreu (2009): "El control político de la información periodística". En: Revista Latina de Comunicación Social, n. 64: 354-366.

ESTEVE, Ramírez y FERNÁNDEZ DEL MORAL, Javier (1995): Áreas de especialización periodística. Fragua. Madrid.

FERNÁNDEZ, Francisco y VILAR, Fernando (2002): "Escenarios presentes y futuros de la prensa local y gratuita". En: La prensa local y la prensa gratuita ComLoc 2001. Publicaciones de la Universitat Jaume I. Servei de Comunicació i Publicacions, 238-239. Castelló.

IZQUIERDO, Luis (2010): Manual de periodismo local. Fragua. Madrid.

LAGUNA, Antonio y MARTÍNEZ, Francesc (1992): Historia de Levante-El Mercantil Valenciano. Editorial Prensa Valenciana, S. A. Valencia. 
LÓPEZ, Xosé (2005): "La prensa local en España. Redescubrir el valor de la proximidad". Revista Telos, 63.

LÓPEZ, Xosé y MACIÀ, Juan (2007): Periodismo de proximidad. Síntesis. Madrid

MARTÍNEZ SANCHIS, Francesc (2010): La informació de proximitat del País Valencià en l'ambit dels territoris de Llengua Catalana. En: Arxius, 23.

MARTÍNEZ SANCHIS, Francesc (2007): "La recerca en comunicació en el País Valenciá. Comunicació local i de proximitat". En: Treballs de Comunicació. Societat Catalana de Comunicació, 22.

MORAGAS, Miquel (1998): "El serveis publics en la societat de la informació. Notes per a una nova interpretació". En: Treballs de Comunicació, 11.

NOS ALDÁS, María Luisa (2001): "Comunicación y sociedad: Propuesta de un modelo de Prensa Local". En: La prensa local y la prensa gratuita ComLoc 2001. Publicaciones de la Universitat Jaume I. Servei de Comunicació i Publicacions, Castelló.

XAMBO, Rafael (2001): Comunicació, política i societat. El cas valencià. Eliseu Climent, editor. Valencia.

Recursos web

FUNDACIÓN BBVA (2013): "Estudio Internacional de la Fundación BBVA. Values and Worldvievs". En: http://fbbva.es/TLFV/teju/esp/home/index.jsp, [29-072013].

LÓPEZ, Xoxé, GALINDO, Fermín y VILLAR, Manuel: "El valor social de la información de proximidad". En: Revista Latina de Comunicación Social, 7. En htttp://www.ull.es/publicaciones/latina/a/68xose.html. [20.06-2013].

DE FONTCUBERTA, Mar (1997): "La identidad regional de los medios". En: Cuadernos de Información, 12. Disponible en: dialnet.unirioja.es/descarga/articulo/2939041.pdf. [17-06-2013].

CALVO, Elvira (2013): http://cultura.elpais.com/cultura/2013/10/13/television/1381689487_208653.html [14 de octubre de 2013].

\section{El autor}

Ramón S. Pardo Baldeón es Licenciado en Ciencias de la Información por la Universidad Complutense de Madrid (1984) y desde septiembre de 2009 Profesor Asociado de Periodismo en la Universitat Jaume I de Castelló. Desde 2008 es vocal de Formación y Nuevas Tecnologías en la Asociación de Periodistas de Castelló. Ha trabajado como redactor en El Comercio, de Gijón (1984-89), en La Nueva España, de Oviedo (199093), y se incorporó a la redacción de Castellón de Levante-El Mercantil Valenciano en marzo de 1993. Ha sido delegado en Gijón de Antena 3 Radio (1982-85) y corresponsal en Gijón de Radio Asturias (SER) entre 1985 y 1992. En 1982 fue responsable de Prensa del Campeonato de Europa de Hockey sobre Patines celebrado en Oviedo. 